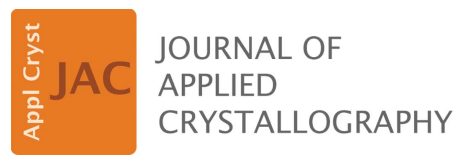

ISSN 1600-5767
Keywords: book reviews; bioelements; interface techniques; bioelectronic materials; bioelectronic sensors and bioelectronic molecular devices

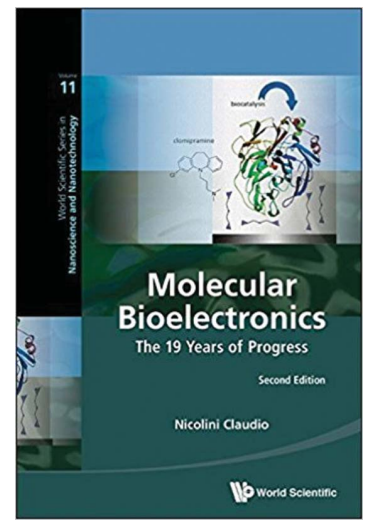

\section{Molecular Bioelectronics. The 19 Years of Progress. Second Edition. By Claudio Nicolini. World Scientific, 2016. Pp. 336. Price GBP 108.00. ISBN 9789814725842.}

\author{
Ashutosh Tiwari* \\ Director, Institute of Advanced Materials, Teknikringen 4A, Mjardevi Science Park, Linkoping 583 30, Sweden. \\ *Correspondence e-mail: director@iaam.se
}

I would like to congratulate Professor Claudio Nicolini for accomplishing the 19 years of progress documented in the second edition of Molecular Bioelectronics, published by World Scientific Publishing, Singapore. This book provides an up-to-date view of the state of the art of various active bioelements ranging from organic compounds, conductive polymers, proteins, lipids and DNA to whole cells and protein microarrays. It overviews the numerous potential applications of proteins, lipids, fatty acids and DNA complexes using different procedures and techniques for thin-film formation, including LangmuirBlodgett and self-assembly processes, manipulation by scanning microscopy at the resolution of a single atom, chemical synthesis, and ab initio calculations for both molecular design and label-free technologies, and further reviews the three-dimensional structure of metalloprotein cytochrome $\mathrm{c}$ and multiple electron transfer pathways. Various bioelectronic materials, for example protein films, thermostable lipid films, fullerene and organic thin films, conductive LB films, and two-dimensional crystals with novel properties like thermal stability and long-range storage, are discussed.

The book gives comprehensive information on the mechanism of bioelectronic sensors utilizing a wide range of transducers, either amperometric, potentiometers, conductivities, spectrophotometric or fluorometric, and assesses emerging bioelectronic sensors for health and environmental applications. The author explains the breakthrough bioelectronic molecular devices like bioactutors, photovoltaic cells, monoelectronic transistors, and DNA- and peptide-sequencing microchips. In the molecular electronics field, molecules are utilized as the active or passive elements in electronic devices. Likewise, the author includes a discussion about the development of circuital elements based on organic materials and biomaterials which have offered an alternative to Si electronics over the past 20 years.

The different chapters with their good referencing allow us to glimpse a new era of molecular bioelectronics. The whole book emphasizes fundamental approaches implemented on biomaterials in the vast field of molecular bioelectronics. In summary, the book studies new bioelectronic structures in depth, as well as the developments achieved in bioelectronic devices over the past 19 years.

I hope that any future edition would consider linking tiny and smart bioelectronics capable of switchable features for new age technology. I would also have expected the author to broaden this topic with more references for the benefit of new researchers. With these additions, this would be a valuable reference book in the field of molecular bioelectronics. 